\title{
Application of linear density response theory to electron-impact autoionizing resonances
}

\author{
Xiao-Min Tong ${ }^{1,2}$ and Tsutomu Watanabe $e^{3,4}$ \\ ${ }^{1}$ Institute of Materials Science, Graduate School of Pure and Applied Sciences, University of Tsukuba, \\ 1-1-1 Tennodai, Tsukuba, Ibaraki 305-8573, Japan \\ ${ }^{2}$ Center for Computational Sciences, University of Tsukuba, 1-1-1 Tennodai, Tsukuba, Ibaraki 305-8577, Japan \\ ${ }^{3}$ Institute for Laser Science, University of Electro-Cominucations, Chofugaoka, Chofu, Tokyo 182-8585, Japan \\ ${ }^{4}$ Department of Physics, Tokyo Metropolitan University, 1-1 Minami-Osawa, Hachioji, Tokyo, 192-0364, Japan
}

(Received 24 September 2007; published 30 October 2007)

\begin{abstract}
We apply the linear density response theory to the electron-impact ionization including autoionizing resonances of atoms. Such a single-electron-like method takes into account the electron-electron dynamic correlation effect through the first-order perturbation theory. The method allows us to study the dynamic correlation in the process of electron-impact excitation with ionization background. We take the autoionizing resonances of Ar atoms as an example to show the simpleness and effectiveness of the present method. Our calculated electron impact ionization cross sections including autoionizing resonances are in reasonable agreement with the experimental measurements.
\end{abstract}

DOI: 10.1103/PhysRevA.76.042715 PACS number(s): 34.80.Dp, 32.80.Dz, 32.70.Cs, 31.15.Ew

\section{INTRODUCTION}

The electron dynamic correlation effects play a fundamental role in atomic and molecular physics $[1,2]$. Atomic photoabsorption spectra of excited discrete levels which are embedded into ionization continuum have been studied by many authors. The dynamic correlation problem in the case of energy loss spectra by charged particle impact is also important and has been studied extensively [3-7]. Unlike the photoabsorption experiments, the electron-impact experiment measures not only the optically allowed transitions but also the optically forbidden transitions. Furthermore, the scattering angle of the incident electron opens another dimension to study the contribution of dynamic correlation in the optically allowed and forbidden transitions. For example, the autoionizing resonance line profile observed by the electron-impact experiment depends not only on the specified transition, but also on the momentum transfer of the incident electron. Thus we can select the resonance line profile tuning the momentum transfer. The dynamical correlation in the autoionizing resonances has been studied by many authors $[1,2,8-11]$ using various theoretical tools, such as the configuration interaction and stabilization method [12], the hyperspherical coordinate method [13], the close-coupling [14], the $R$-matrix theory [15], and others. Here we only quote the most recent paper for respective method. Concerning these documents, a more detailed list can be found in the references in a paper by Yuan et al. [16]. However, most of the above papers treated only resonant energy positions and resonance widths and did not pay attention to the energy loss spectra and the momentum transfer dependence. Recently, the detailed energy loss spectra for He atoms have been measured [17] and the relevant calculations have been performed [16] using the $R$-matrix theory. In principle, the electronimpact autoionizing resonance can be studied by the $R$-matrix theory for many-electron systems as well. The computational effort will increase dramatically as the number of electrons increases. Thus, we would like to find a simple, yet effective method to study the electron-impact autoionizing resonance.
The linear density response theory combined with the self-interaction free density function theory has been used successfully to study the autoionizing resonances [18] and giant resonances $[19,20]$ in the photoabsorption processes. This is a simple single-electron-like theory in which the electron-electron dynamic correlation is taken into account through the first-order perturbation theory. The computational effort increases linearly as the number of electrons increases. We extend the method to study the energy loss spectra of incident charged particle as a function of the scattering angle or momentum transfer. Here we use an optimized effective potential and incorporate a self-interaction correction. We take the electron-impact autoionizing resonance of Ar atoms as an example to show the effectiveness of the method. Argon is one of the most basic species in the problem. Fano $[1,2]$ has studied the Ar photoabsorption spectra, which were experimentally observed by Madden et al. [4] at the early stage of this type of research. Burke and Taylor [21] calculated the photoexcitation of inner-valence $3 s$ electron of Ar atoms using the $R$-matrix method. Recently Wu et al. [22] measured the energy-loss spectra of the highenergy electron impact on Ar atoms in the energy losses around $25-31 \mathrm{eV}$, which covers the excitations from the $3 \mathrm{~s}$ electron to the $n p, n d$, and $n s$ Rydberg series below the $3 s\left(3 p^{6}\right)\left({ }^{2} S\right)$ ionization threshold at different scattering angles. Additionally a Japanese group [23] has also measured the energy loss spectra using a relative lower incident electron beam. Thus, the electron-impact autoionizing resonance of Ar atoms serves as a good candidate to test our method. In the following, we describe the detailed theoretical method in Sec. II and present our results and the comparison with the experiments in Sec. III.

\section{THEORETICAL METHOD}

Based on the Born approximation, the electron-impact excitation or ionization can be studied by the generalized oscillator strength (GOS), which is define as (atomic units $e$ $=\hbar=m=1$ are used unless otherwise specified) 


$$
f(\Delta E, \mathbf{Q})=\frac{2 \Delta E}{Q^{2}}\left|\left\langle\Psi_{f}\left|\sum_{j} e^{i \mathbf{Q} \cdot \mathbf{r}_{j}}\right| \Psi_{0}\right\rangle\right|^{2} .
$$

Here, $\Psi_{0}$ and $\Psi_{f}$ are the initial and final states of the target atoms with energies $E_{0}$ and $E_{f}, \Delta E$, and $\mathbf{Q}$ are the impact electron energy loss and momentum transfer during the collision and summation $j$ runs over all the electrons of the target atom. For a many-electron system, the calculation of the high precision correlated wave functions $\Psi_{0}$ and $\Psi_{f}$ is still a challenge to the atomic structure studies, especially for the final state $\Psi_{f}$, which contains both discrete and continuum states. With the independent particle approximation (IPA), the above equation can be simplified as

$$
f(\Delta E, \mathbf{Q})=\left.\frac{2 \Delta E}{Q^{2}} \sum_{i, j} n_{i}\left(1-n_{j}\right)\left|\left\langle\psi_{j}\right| e^{i} \mathbf{Q} \cdot \mathbf{r}\right]\left|\psi_{i}\right\rangle\right|^{2} .
$$

Here $\psi_{i}$ and $\psi_{j}$ are the initial and final states of the single electron transition with corresponding energies $\epsilon_{i}$ and $\epsilon_{j}$, and occupation numbers $n_{i}$ and $n_{j}$. The calculation of Eq. (2) is straightforward and it can be used to study the electronimpact excitation or ionization processes. Unfortunately, it cannot be used to study the autoionizing resonances because the interaction between the discrete and continuum states are completely ignored in the above equation.

The linear density response theory $[24,25]$ has been successfully used to study the dynamic correlation in the photoionization processes. It takes the simplicity of the IPA while taking into account the dynamic correlation effect. We will extend the linear density response theory to the study of electron-impact autoionizing resonances. According to the linear density response theory, the frequency-dependent induced electron density $\delta \rho(\mathbf{r}, \omega)$ by the external potential $V^{\text {ext }}(\mathbf{r}, \omega)$ can be expressed as

$$
\delta \rho(\mathbf{r}, \omega)=\int \chi\left(\mathbf{r}, \mathbf{r}^{\prime}, \omega\right) V^{\mathrm{ext}}\left(\mathbf{r}^{\prime}, \omega\right) d^{3} \mathbf{r}^{\prime} .
$$

For the GOS calculation, the external potential is

$$
V^{\mathrm{ext}}(\mathbf{r}, \omega)=e^{i \mathbf{Q} \cdot \mathbf{r}}=\sum_{k m} 4 \pi i^{k} j_{k}(Q r) Y_{k m}^{*}(\hat{\mathbf{Q}}) Y_{k m}(\hat{\mathbf{r}}) .
$$

The susceptibility $\chi\left(\mathbf{r}, \mathbf{r}^{\prime}, \omega\right)$ can be determined by means of the first-order time-dependent perturbation theory [25] and expressed in terms of the eigenfunctions $\left\{\psi_{i}\right\}$ and the eigenvalues $\left\{\epsilon_{i}\right\}$ as

$$
\chi^{\mathrm{IPA}}\left(\mathbf{r}, \mathbf{r}^{\prime}, \omega\right)=\sum_{i, j}\left(n_{i}-n_{j}\right) \frac{\psi_{i}^{*}(\mathbf{r}) \psi_{j}(\mathbf{r}) \psi_{i}\left(\mathbf{r}^{\prime}\right) \psi_{j}^{*}\left(\mathbf{r}^{\prime}\right)}{\omega-\left(\epsilon_{j}-\epsilon_{j}\right)+i \eta},
$$

where $\eta$ is a positive infinitesimal used to ensure the outgoing wave boundary conditions. $\left\{\psi_{i}(\mathbf{r})\right\}$ and $\left\{\epsilon_{i}\right\}$ are calculated from the Schrödinger equation,

$$
\left[-\frac{1}{2} \nabla^{2}+V^{\mathrm{eff}}(r)\right] \psi_{i}(\mathbf{r})=\epsilon_{i} \psi_{i}(\mathbf{r}),
$$

where $V^{\text {eff }}(r)$ is an effective potential which is obtained from the optimized effective potential method [26]. Under the self-consistent-field approximation, the electron density change $\delta \rho(\mathbf{r}, \omega)$ is rewritten as

$$
\delta \rho(\mathbf{r}, \omega)=\int \chi^{\mathrm{IPA}}\left(\mathbf{r}, \mathbf{r}^{\prime}, \omega\right) V^{\mathrm{SCF}}\left(\mathbf{r}^{\prime}, \omega\right) d \mathbf{r}^{\prime},
$$

where $V^{\mathrm{SCF}}(\mathbf{r}, \omega)$ is obtained by the following equation:

$$
\begin{aligned}
V^{\mathrm{SCF}}(\mathbf{r}, \omega)= & V^{\mathrm{ext}}(\mathbf{r}, \omega)+\int \frac{\delta \rho(\mathbf{r}, \omega)}{\left|\mathbf{r}-\mathbf{r}^{\prime}\right|} d \mathbf{r}^{\prime} \\
& +\left.\frac{\partial V_{x c}(\mathbf{r})}{\partial \rho(\mathbf{r})}\right|_{\rho_{0}(\mathbf{r})} \delta \rho(\mathbf{r}, \omega) .
\end{aligned}
$$

Here $V_{x c}(\mathbf{r})$ is the exchange correlation potential and $\rho_{0}(\mathbf{r})$ is the ground-state electron density. We calculate $\delta \rho(\mathbf{r}, \omega)$ and $V^{\mathrm{SCF}}(\mathbf{r}, \omega)$ solving Eqs. (7) and (8) iteratively. Detailed procedure has been shown in our previous work [19]. With the obtained $\delta \rho(\mathbf{r}, \omega)$, the GOS is expressed as

$$
f(\Delta E, Q)=\frac{2 \Delta E}{Q^{2}} \operatorname{Im} \int V^{\mathrm{ext}}(\mathbf{r}, \omega) \delta \rho(\mathbf{r}, \omega) d \mathbf{r} .
$$

Due to the parity conservation and the angular-momentum conservation, we can decompose each $k$ th pole contributions by replacing $V^{\text {ext }}(\mathbf{r}, \omega)$ with

$$
V_{k}^{\mathrm{ext}}(\mathbf{r}, \omega)=\sum_{m} 4 \pi i^{k} j_{k}(Q r) Y_{k m}^{*}(\hat{\mathbf{Q}}) Y_{k m}(\hat{\mathbf{r}})
$$

in Eqs. (7) and (8) and obtain the corresponding GOS labelled as $f^{k}(\Delta E, Q)$. Thus, the total GOS can be rewritten as

$$
f(\Delta E, Q)=\sum f^{k}(\Delta E, Q) .
$$

With the calculated GOS, the electron-impact excitation or ionization cross sections are obtained as

$$
\sigma(\omega)=\frac{2 \pi}{T} \frac{1}{\Delta E} \int_{Q_{\min }}^{Q_{\max }} \frac{f(\Delta E, Q)}{Q} d \mathbf{Q},
$$

with $T$ the incident electron energy and $Q_{\min }\left(Q_{\max }\right)$ the minimum (maximum) momentum transfer.

Note that in the above equations the electron energy loss $\Delta E$ and the transition energy $\omega$ are equal to each other due to the energy conservation. The above equations are derived for the spin-paired system. It is straightforward to extend them to the spin-unpaired system as we did for the photoionization studies [19].

\section{ELECTRON IMPACT AUTOIONIZING RESONANCES OF Ar ATOMS}

Autoionizing resonances of Ar atoms below the $3 s$ ionization threshold have been studied extensively by the photoionization or the electron-impact ionization both experimentally [27-30] and theoretically [31]. We would like to take the electron-impact autoionizing resonances of Ar atoms as an example to show the effectiveness of our present method. The GOS formula was derived under the Born approximation so it should be valid for the high-energy electron-impact experiment. Wu et al. [22] have measured the GOS using a $2.5 \mathrm{keV}$ electron beam at scattering angles $0^{\circ}$ and $2^{\circ}$. For the $0^{\circ}$ scattering angle, the measured spectra approach to the 


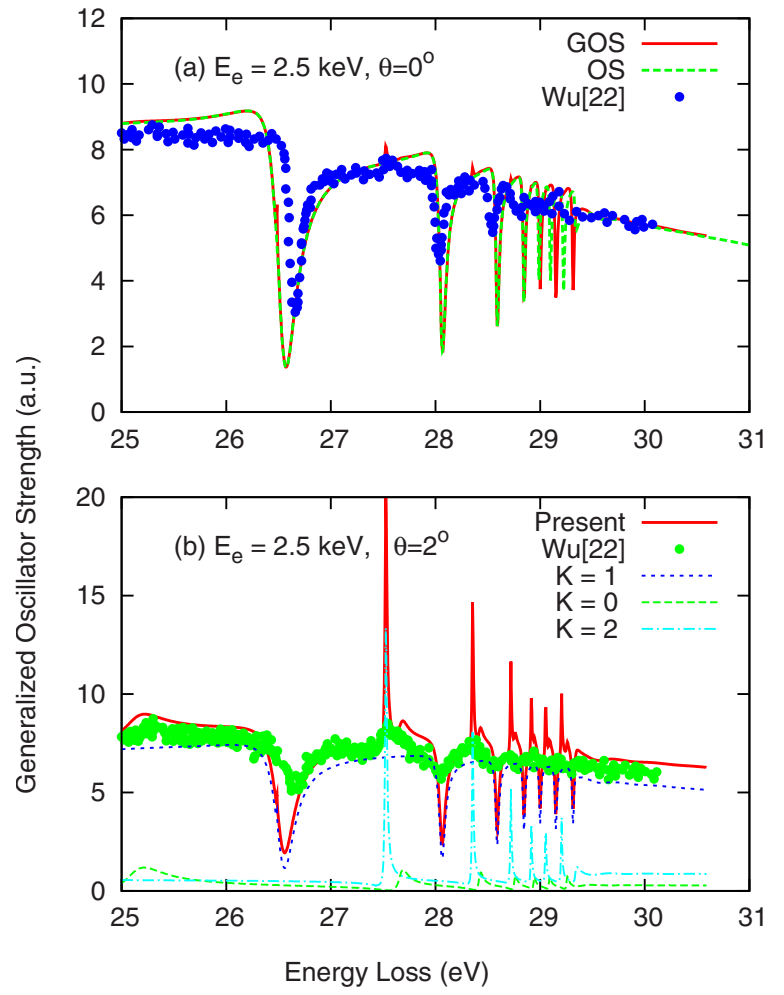

FIG. 1. (Color online) The generalized oscillator strengths of $\mathrm{Ar}$ atoms as a function of the energy loss by a $2.5 \mathrm{keV}$ electron beam with scattering angles (a) $\theta=0^{\circ}$ and (b) $\theta=2^{\circ}$. The filled circles are from experiment [22].

oscillator strength, which corresponds to the photoabsorption spectra. We first compare our results with their measurement as shown in Fig. 1. For scattering angle $\theta=0^{\circ}$, the excitations from $3 s$ to $n p$ series form diplike resonances as shown in Fig. 1(a). For the lowest resonances, namely $4 p$ and $5 p$, our results are in agreement with the measured ones in the absolute value. Due to the experimental energy resolution, they did not observe the narrow resonances of the highly excited states, which show up in our simulations. The zero degree scattering spectra is a mimic of the photoabsorption spectra since the GOS goes to the optical oscillator strength as $Q$ approaches zero. In the simulation, since $Q$ is a finite value, we still observe a tiny contribution from the excitation of $3 \mathrm{~s}$ to $n d$ series as shown in Fig. 1(a). The tiny peak around $27.5 \mathrm{eV}$ in the experimental spectra could come from the $3 \mathrm{~s}$ to $3 d$ transition. Such a transition is forbidden by the dipole selection rule and it does not appear in the photoabsorption spectra by synchrotron radiation [4].

As for scattering angle $\theta=2^{\circ}$, the optical forbidden transitions, namely, $3 s$ to $n s$ and $n d$ series, appear with sharp resonances. To identify the measured resonance, we also plot $f^{k}(\Delta E, Q)$ for $k=0,1,2$ in Fig. 1(b). Comparing our results with the experimental ones, we identify that the observed peak around $27.5 \mathrm{eV}$ is attributed to the $3 s$ to $3 d$ transition with a minor contribution from $3 s$ to $4 s$. Note that we scale the experimental results, which were presented by arbitrary units in their original paper [22], to our results in absolute value. Overall, our results are in reasonable agreement with the experimental ones. The discrepancies mainly come from

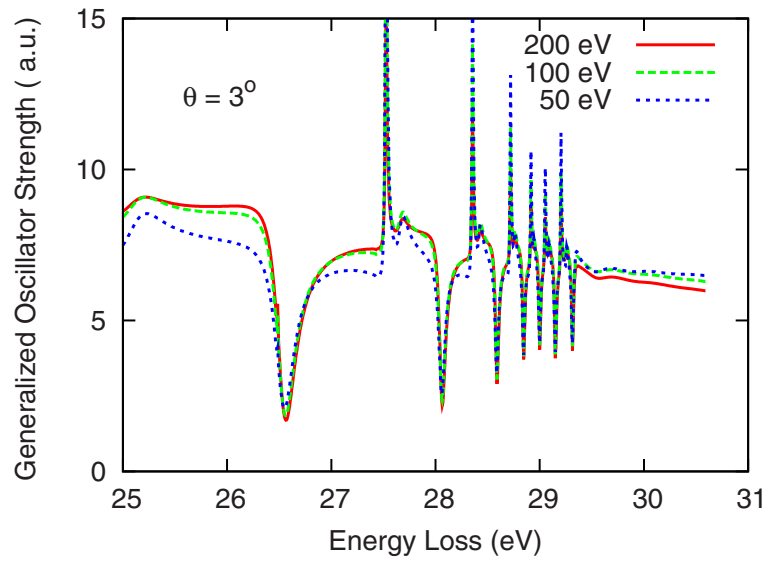

FIG. 2. (Color online) The generalized oscillator strengths of Ar atoms as a function of the energy loss by fixed scattering angle $\theta$ $=3^{\circ}$ with different incident energies.

the experiment energy resolution, which we do not take into account in our simulation. To compare with the measured spectra, we shift our calculated energy loss by $0.9 \mathrm{eV}$, which is originated from the energy difference between our calculated $3 s$ ionization potential and the measured one.

The GOS is only valid to describe the electron-impact excitation or ionization processes for the high-energy electron beam. Now we want to know what is the criterion for the high energy. Figure 2 shows the GOS of Ar atoms with a fixed scattering angle at $\theta=3^{\circ}$ with 200,100 , and $50 \mathrm{eV}$ incident electron energies, respectively. Our simulation shows that GOS in the near forward direction is not sensitive to the incident energy when the incident energy is above $100 \mathrm{eV}$ in the present case. Here we calculate the GOS with the $Q$ value corresponding to the momentum transfer. We need to compare the relevant experiments to check if the Born approximation is valid for the lower incident energies. Fortunately, the Japanese group [23] has performed such an experiment recently. Comparing with their results, we find that our results of autoionizing resonance are in reasonable agreement for the incident energy above $100 \mathrm{eV}$. For the lower incident energy, namely $50 \mathrm{eV}$ or even lower, such as $35 \mathrm{eV}$, our results differ from the experimental ones significantly. Thus we conclude that the Born approximation could be valid for the incident electron energy above $100 \mathrm{eV}$ in the present case, which is about 3 times of the $3 s$ ionization threshold.

When we calculate the GOS as a function of the scattering angle $\theta$ we find that the GOS is not very sensitive to the scattering angle for the lower incident energy. We plot the GOS of the scattering angles from $0^{\circ}$ to $2^{\circ}$ with 2500,500 , and $100 \mathrm{eV}$ incident energies in Fig. 3. The GOS changes largely for the higher incident energy (between the two solid curves for $2500 \mathrm{eV}$ ) than that of the lower incident energy (between the two dotted curves for $100 \mathrm{eV}$ ) if we fix the scattering angular resolution. This can be easily understood since the momentum transfer $Q$ strikes into the scattering angle $\theta$ by the following equation:

$$
Q^{2}=k_{i}^{2}+k_{f}^{2}-2 k_{i} k_{f} \cos \theta
$$

with $k_{i}$ and $k_{f}$ the initial and final momenta of the incident electron. For the high incident energy, the finite scattering 


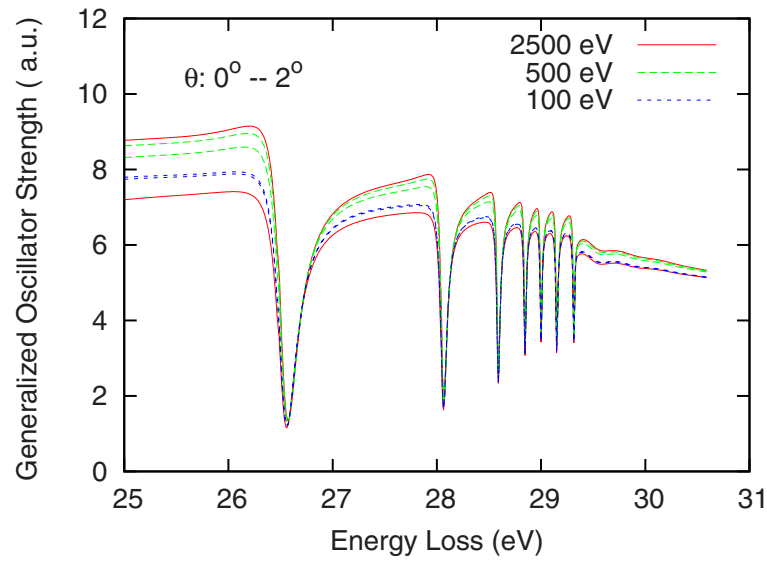

FIG. 3. (Color online) The dipole-allowed transition generalized oscillator strengths of Ar atoms as a function of the energy loss for scattering angles from $0^{\circ}$ to $2^{\circ}$ with different incident energies.

angular resolution covers a large range of $Q$, which leads to the large uncertainty in the observed GOS. The relative lower energy experiment will have better resolution with a limited $Q$ range. Therefore, we must balance the resolution and the $Q$ range in experiments.

The most interesting part in the GOS is the autoionizing structure. Due to the interaction between the discrete and continuum states, the resonance peak is of the Fano profile. Figure 4 shows the line profiles of the transition from $3 s$ to $4 p$ as a function of the momentum transfer $Q$. Clearly we see that the GOS decreases as the $Q$ value increases. Meanwhile the shape of the GOS also changes from a diplike resonance to a peaklike resonance as the $Q$ value increases. The resonance line profile can be described by the Fano profile [2] as

$$
f(\Delta E, Q)=f_{0}\left(\rho^{2} \frac{(q+x)^{2}}{1+x^{2}}-\rho^{2}+1\right),
$$

with

$$
x=\frac{\Delta E-E_{r}}{\Gamma / 2} .
$$

Here $f_{0}$ is the GOS, $q$ is the line profile index, $E_{r}$ is the resonance energy position, $\Gamma$ is the resonance half-width, and

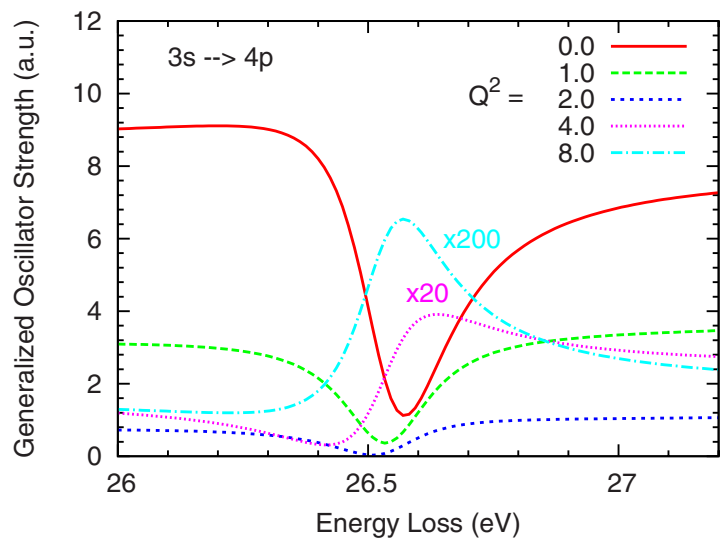

FIG. 4. (Color online) The line profiles of the generalized oscillator strength of Ar atoms near the $3 s$ to $4 p$ transition for different momentum transfers.

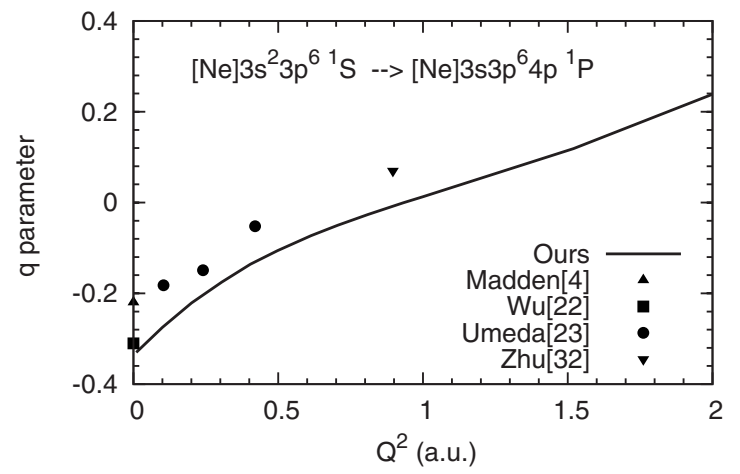

FIG. 5. The Fano $q$ parameters of $3 s$ to $4 p$ autoionizing resonance as a function of the momentum transfer $Q$.

$\rho^{2}$ is the correlation coefficient, which gives the proportion of the continuum that interacts with the autoionizing states. The $q$ parameter describes the resonance spectra pattern, so we plot the $q$ parameter as a function of the momentum transfer $Q$ in Fig. 5. We also plot the available experimental data in the figure. Physically speaking, the Fano $q$-parameter can be expressed as

$$
q=\frac{\left\langle\Psi_{0}\left|V^{\mathrm{ext}}\right| \Psi_{c}\right\rangle}{\left\langle\Psi_{0}\left|V^{\mathrm{ext}}\right| \Psi_{b}\right\rangle}
$$

Here $\Psi_{0}, \Psi_{c}, \Psi_{b}$ are the initial, final discrete, and final continuum wave functions and $V^{\text {ext }} \propto j_{1}(Q r)$. The initial and excitation wave functions are localized in a small region and the transition matrix element $\left\langle\Psi_{0}\left|V^{\text {ext }}\right| \Psi_{b}\right\rangle$ is not sensitive to the changes of the $Q$ value. The transition matrix element $\left\langle\Psi_{0}\left|V^{\text {ext }}\right| \Psi_{c}\right\rangle$ is sensitive to the changes of the $Q$ value. This is only a qualitative analysis. A quantitative calculation is difficult since we do not know the $\Psi_{c}, \Psi_{b}$. We see that the $q$ parameter increases from a negative value to zero as $Q$ increases from 0 to 1 , and then to a positive value as $Q$ increases further. This trend is consistent with the experimental observations [22,23,32] although our $q$ parameters are smaller than the experimental ones. The discrepancies between our results and the experimental ones may come from the high-order interactions which are ignored in the present simple method. They may also come from the finite resolution of the experiments, which has been studied by Fang and Chang [33]. One bound interacting with several continua [34] may also contribute to the discrepancies. The discrepancies call for a further study of the problem by more sophisticated theoretical methods.

To summarize, we applied the linear density response theory to the study of electron-impact autoionizing resonances of Ar atoms below the $3 s$ ionization threshold. Our results are in good agreement with the available experiments including the autoionizing resonance shapes. Furthermore, we predicted that the resonance shape of the $3 s$ to $4 p$ transition changes from a diplike resonance to a peaklike resonance as the momentum transfer $Q$ increases. A recent experiment by the Japanese group partly confirmed this prediction and we hope further systematic experiment can test the prediction. 


\section{ACKNOWLEDGMENTS}

This research was partially supported by Grand-in-Aid for Scientific Research (C) from the Japan Society for the Pro- motion of Science. The authors would like to thank Professor Y. Sakai, Professor N. Nakamura, and Professor S. Ohtani for providing their experimental data as well as for their stimulating discussions.
[1] U. Fano, Phys. Rev. 124, 1866 (1961).

[2] U. Fano and J. W. Cooper, Phys. Rev. 137, A1364 (1965).

[3] J. A. Simpson, G. E. Chamberlain, and S. R. Mielczarek, Phys. Rev. 139, A1039 (1965).

[4] R. P. Madden, D. L. Ederer, and K. Codling, Phys. Rev. 177, 136 (1969).

[5] W. F. Chan, G. Cooper, and C. E. Brion, Phys. Rev. A 44, 186 (1991).

[6] S. J. Brotton, S. Cvejanovic, F. J. Currell, N. J. Bowring, and F. H. Read, Phys. Rev. A 55, 318 (1997).

[7] X. W. Fan and K. T. Leung, J. Phys. B 34, 811 (2001).

[8] P. G. Burke and D. D. McVicar, Proc. Phys. Soc. London 86, 989 (1965)

[9] B. W. Shore, Rev. Mod. Phys. 39, 439 (1967).

[10] B. W. Shore, Phys. Rev. 171, 43 (1968).

[11] F. H. Mies, Phys. Rev. 175, 164 (1968).

[12] M. Venuti, P. Decleva, and A. Lisini, J. Phys. B 29, 5315 (1996).

[13] T. Morishita, K. I. Hino, S. Watanabe, and M. Matsuzawa, Phys. Rev. A 53, 2345 (1996).

[14] S. Salomonson, S. L. Carter, and H. P. Kelly, Phys. Rev. A 39, 5111 (1989).

[15] T. Schneider, C. N. Liu, and J. M. Rost, Phys. Rev. A 65, 042715 (2002).

[16] Z. S. Yuan, X. Y. Han, X. J. Liu, L. F. Zhu, K. Z. Xu, L. Voky, and J. M. Li, Phys. Rev. A 70, 062706 (2004).

[17] X. J. Liu, L. F. Zhu, Z. S. Yuan, W. B. Li, H. D. Cheng, Y. P. Huang, Z. P. Zhong, K. Z. Xu, and J. M. Li, Phys. Rev. Lett.
91, 193203 (2003).

[18] X. M. Tong and S. I. Chu, Chem. Phys. 217, 119 (1997).

[19] X. M. Tong, D. Kato, T. Watanabe, and S. Ohtani, J. Phys. B 33, 717 (2000).

[20] X. M. Tong, D. Kato, T. Watanabe, and S. Ohtani, Phys. Rev. A 64, 032716 (2001)

[21] P. G. Burke and K. T. Taylor, J. Phys. B 8, 2620 (1975).

[22] S. L. Wu, Z. P. Zhong, R. F. Feng, S. L. Xing, B. X. Yang, and K. Z. Xu, Phys. Rev. A 51, 4494 (1995).

[23] N. Umeda, Y. Sakai, T. Takayanagi, N. Nakamura, C. Yamada, and S. Ohtani (unpublished).

[24] R. Kubo, J. Phys. Soc. Jpn. 12, 578 (1957).

[25] A. Fetter and J. Walecka, Quantum Theory of Many-Body Systems (McGraw-Hill, New York, 1971).

[26] X. M. Tong and Shih-I. Chu, Phys. Rev. A 55, 3406 (1997).

[27] K. H. Schartner, B. Möbus, P. Lenz, H. Schmoranzer, and M. Wildberger, Phys. Rev. Lett. 61, 2744 (1988).

[28] X. W. Fan and K. T. Leung, Phys. Rev. A 62, 062703 (2000).

[29] A. A. Sorokin, L. A. Shmaenok, S. V. Bobashev, B. Möbus, M. Richter, and G. Ulm, Phys. Rev. A 61, 022723 (2000).

[30] H. D. Cheng, L. F. Zhu, X. J. Liu, Z. S. Yuan, W. B. Li, and K. Z. Xu, Phys. Rev. A 71, 032714 (2005).

[31] F. A. Parpia, W. R. Johnson, and V. Radojevic, Phys. Rev. A 29, 3173 (1984).

[32] L. F. Zhu, H. D. Cheng, X. J. Liu, P. Tian, Z. S. Yuan, W. B. Li, and K. Z. Xu, Chin. Phys. Lett. 20, 1718 (2003).

[33] T. K. Fang and T. N. Chang, Phys. Rev. A 57, 4407 (1998).

[34] P. Durand and I. Paidarová, J. Phys. B 35, 469 (2002). 